\title{
COMPLICATIONS OF PANCREATITIS- CT EVALUATION
}

\author{
Anjali Pravinkumar Wasadikar1, Vijay Devji Wakode2, Varsha Jagannath Rote-Kaginalkar³, Pravinkumar Prabhakar Wasadikar4, \\ Anjali Pawar-Dahiphale5, Kavita Ramrao Makasare ${ }^{6}$ \\ ${ }_{1}^{1}$ Associate Professor, Department of Radiodiagnosis, Government Medical College and Hospital, Aurangabad, Maharashtra. \\ 23rd Year Junior Resident, Department of Radiodiagnosis, Government Medical College and Hospital, Aurangabad, Maharashtra. \\ 3Professor and HOD, Department of Radiodiagnosis, Government Medical College and Hospital, Aurangabad, Maharashtra. \\ ${ }^{4}$ Associate Professor, Department of General Surgery, Government Medical College and Hospital, Aurangabad, Maharashtra. \\ ${ }^{5}$ Associate Professor, Department of Radiodiagnosis, Government Medical College and Hospital, Aurangabad, Maharashtra. \\ ${ }^{6}$ Assistant Professor, Department of Radiodiagnosis, Government Medical College and Hospital, Aurangabad, Maharashtra.
}

\section{BACKGROUND}

ABSTRACT

Acute pancreatitis is a common condition with variable clinical course. Imaging studies play an important role in diagnosis and management of acute pancreatitis. Computed Tomography (CT) is undertaken to determine the role of CT in acute pancreatitis, to differentiate between oedematous and necrotising pancreatitis and to evaluate the complications and severity using Modified Computed Tomography Severity Index (MCTSI).

\section{MATERIALS AND METHODS}

This is a prospective observational study. Sixty patients with clinical suspicion of acute pancreatitis underwent contrast enhanced CT during two years period. MCTSI score for acute pancreatitis was calculated which includes assessment of pancreatic inflammation, necrosis and extrapancreatic complications.

\section{RESULTS}

Peripancreatic fat stranding was the common feature seen in $90 \%$ patients. Extrapancreatic complications were noted in 32 (53\%) patients and pancreatic necrosis in $20(33 \%)$ patients.

\section{CONCLUSION}

CECT of abdomen in acute pancreatitis helps in differentiating between oedematous and necrotising pancreatitis. The MCTSI helps in evaluating the percentage of pancreatic necrosis and to predict the possibility of developing local and systemic complications. Depending upon the MCTSI grading, the treatment plan can be implemented more effectively and accurately.

\section{KEYWORDS}

Acute pancreatitis, Computed Tomography, Modified Computed Tomography Severity Index (MCTSI), Contrast Enhanced Computed Tomography (CECT).

HOW TO CITE THIS ARTICLE: Wasadikar AP, Wakode VD, Kaginalkar VJR, et al. Complications of pancreatitis- CT evaluation. J. Evolution Med. Dent. Sci. 2018;7(14):1796-1800, DOI: 10.14260/jemds/2018/405

\section{BACKGROUND}

Acute pancreatitis is a disease with high morbidity and mortality with an unpredictable course. It has a broad spectrum that varies from mild-to-severe forms with significant local and systemic complications. Severe pancreatitis is characterised by a protracted clinical course, multiorgan failure and pancreatic necrosis. The majority of patients with mild disease recover completely, approximately $15 \%-20 \%$ of patients develop clinically severe AP with local and systemic complications with high mortality.[1] CT is the gold standard non-invasive method of evaluating the morphology of pancreas and peripancreatic regions. It is unaffected by bowel gas distension and obesity, which is a definite disadvantage on ultrasonographic evaluation.

'Financial or Other Competing Interest': None.

Submission 29-01-2018, Peer Review 15-03-2018,

Acceptance 21-03-2018, Published 02-04-2018.

Corresponding Author:

Dr. Pravinkumar Prabhakar Wasadikar,

\#39, Ulkanagari, Near Onkareshwar Mandir,

Garkheda Area,

Aurangabad-431005,

Maharashtra, India.

E-mail: anjailpravinpo@gmail.com

DOI: $10.14260 /$ jemds $/ 2018 / 405$
CECT helps in early diagnosis and staging of severity of acute pancreatitis and its complications, which helps in prediction of prognosis of the disease.

Severity stratification is important during the initial work-up of acute pancreatitis cases. Various CT scoring systems are available. CT Severity Index developed by Balthazar[2] and MCTSI by Mortele et al,[3] total score in both the CT scoring systems is 10 points. Computed Tomography scan with intravenous (IV) contrast agent is required to determine the presence and extent of pancreatic necrosis as well as inflammatory changes, local or extrapancreatic complications. The aim of this study was to determine the role of computed tomography (CECT) in early diagnosis of acute pancreatitis and evaluate the complication using Modified computed tomography severity index MCTSI to assess the severity of AP.

\section{MATERIALS AND METHODS}

This prospective observational study was carried out in 60 patients over a period of two years with permission of Institutional Ethical Committee. Patients with clinical suspicion of acute pancreatitis and those diagnosed as pancreatitis on sonography underwent CECT scan. 


\section{Inclusion Criteria}

1. All the patients who are suspected of acute pancreatitis based on clinical findings.

2. Patients who are diagnosed of acute pancreatitis on ultrasonography.

3. Patients who present as acute on chronic pancreatitis.

\section{Exclusion Criteria}

1. Suspected acute pancreatitis patients with normal pancreas on CT scan.

2. Deranged renal function test.

3. Patient with sensitivity to iodinated contrast media.

Examinations were performed on GE LIGHTSPEED VCT (VOLUME CT) SCANNER 64-SLICE CT. CT examination was done after written and informed consent. Contrast enhanced scans were obtained after intravenous injection of $80 \mathrm{~mL}$ of non-ionic contrast at the rate of $2.5 \mathrm{~mL} / \mathrm{sec}$. Opacification and distension of bowel loops and stomach was achieved by oral administration of $750 \mathrm{~mL}$ of diluted iodinated contrast. Scan protocol used for pancreas evaluation was as follows: Field of Vision (FOV) extending from dome of diaphragm upto inferior border of pubic symphysis. Triphase scans were obtained, scan delay for arterial phase $20 \mathrm{sec}$, venous phase $60 \mathrm{sec}$ and pancreatic parenchymal phase $35 \mathrm{sec}$ was used. Slice thickness of $8 \mathrm{~mm}$ and reconstruction interval of $2.5 \mathrm{sec}$ was used.

\begin{tabular}{|lc|}
\hline \multicolumn{2}{|l|}{ The Modified CT Severity Index (MCTSI) was calculated as } \\
CT Score & Points \\
Normal pancreas & 0 \\
Inflammation - pancreas and/or peripancreatic fat & 2 \\
Pancreatic or peripancreatic fluid collection & 4 \\
Percentage of pancreatic necrosis & \\
None & 0 \\
$\leq 30 \%$ & 2 \\
$>30 \%$ & 4 \\
Extrapancreatic complications & \\
Pleural effusion, ascites, vascular complication, \\
extrapancreatic parenchymal abnormalities \\
or GI tract involvement
\end{tabular}

Modified severity index $=$ CT score + percentage necrosis + extrapancreatic complications.

\begin{tabular}{|lc|}
\hline -Mild & (Points) \\
& $(0-2)$ \\
-Moderate & $(4-6)$ \\
-Severe & $(8-10)$ \\
\hline
\end{tabular}

RESULTS

\begin{tabular}{|c|c|c|c|}
\hline \multirow{4}{*}{ Gland } & CT Findings & Number & \% \\
\hline & Normal size & 13 & 21.6 \\
\cline { 2 - 4 } & $\begin{array}{c}\text { Diffuse } \\
\text { enlargement }\end{array}$ & 25 & 41.6 \\
\cline { 2 - 4 } & $\begin{array}{c}\text { Focal } \\
\text { enlargement }\end{array}$ & 22 & 36.6 \\
\hline Oedematous & & 15 & 25 \\
\hline \multirow{2}{*}{ Necrosis } & $\leq 30 \%$ & 16 & 26.6 \\
\cline { 2 - 4 } & $>30 \%$ & 04 & 6.6 \\
\hline Peripancreatic fat stranding & 54 & 90 \\
\hline \multicolumn{2}{|c|}{ Peripancreatic fluid } \\
collection
\end{tabular}

\begin{tabular}{|c|c|c|}
\hline MCTSI Scores & Pancreatic Necrosis (n= 20) & \% \\
\hline Mild (2 and 4) & 4 & 20 \\
\hline Moderate (6) & 4 & 20 \\
\hline $\begin{array}{c}\text { Severe (8 and } \\
\text { 10) }\end{array}$ & 12 & 60 \\
\hline \multicolumn{2}{|c|}{ Table 2. Distribution of Pancreatic Necrosis according } \\
to MCTSI Scores \\
\hline
\end{tabular}

Focal enlargement was observed in 22 patients with head and neck region in 18 patients being commonest. Commonest site of fluid collection and pseudocyst formation was lesser sac. The extrapancreatic complications were ascites in 24 patients, pleural effusion in 19 , splenic vein thrombosis in seven, portal vein thrombosis in three and pancreaticopleural fistula in one patient. We observed mild pancreatitis in 29 patients, moderate in 18 and severe in 13 patients according to MCTSI score. Patients with severe pancreatitis required intensive care management.

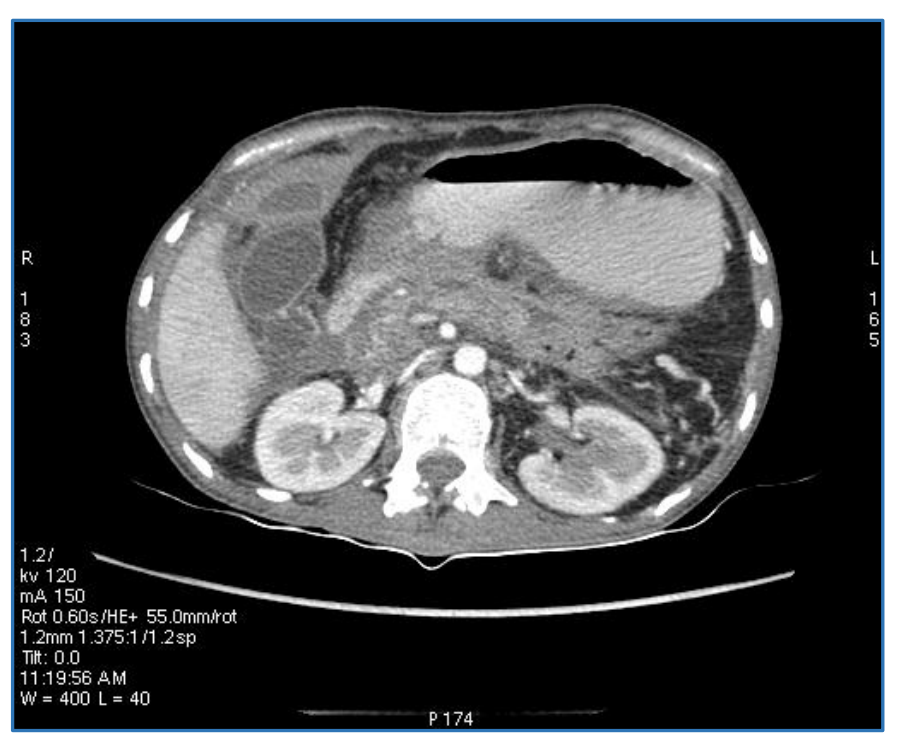

Figure 1. Diffuse Non-Enhancing Area involving Pancreas with adjacent Fat Stranding around Pancreas and Right Paracolic Region suggestive of Pancreatic Necrosis 


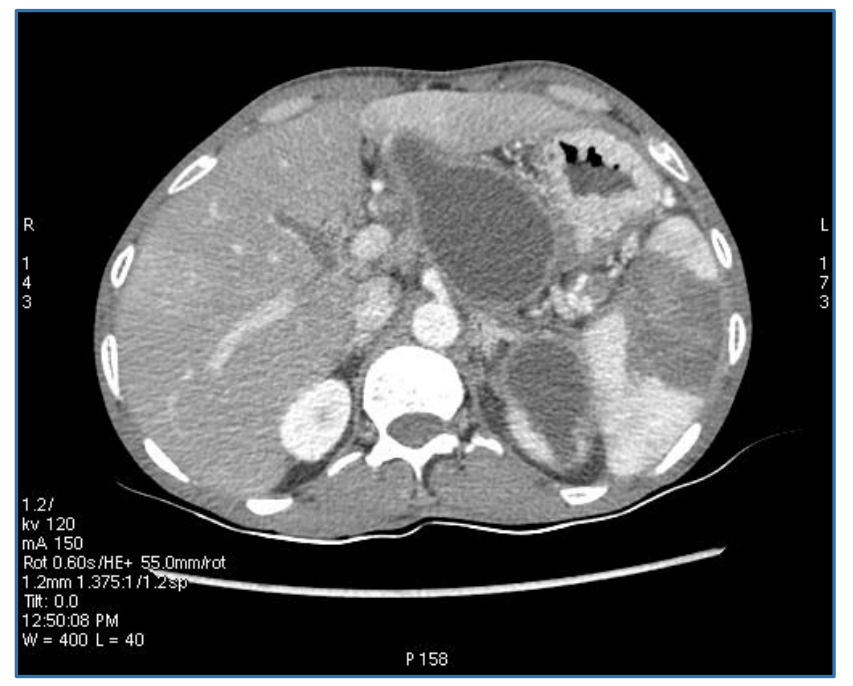

Figure 2. Axial Image showing Pancreatic Pseudocyst Formation with Splenic Vein Thrombosis and Infarct

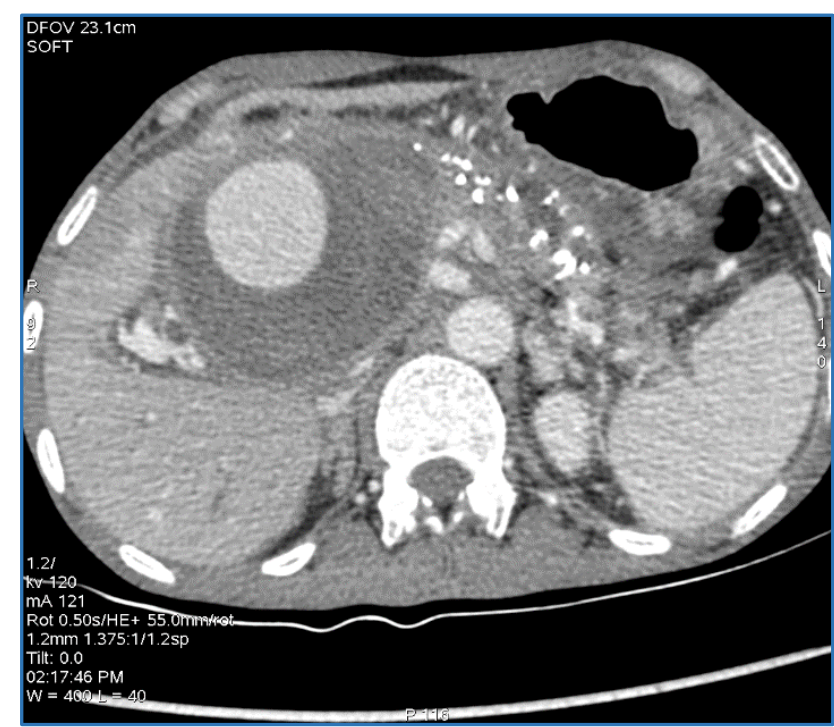

Figure 3. Pseudoaneurysm Formation with Thrombosis arising from the Branch of Right Hepatic Artery

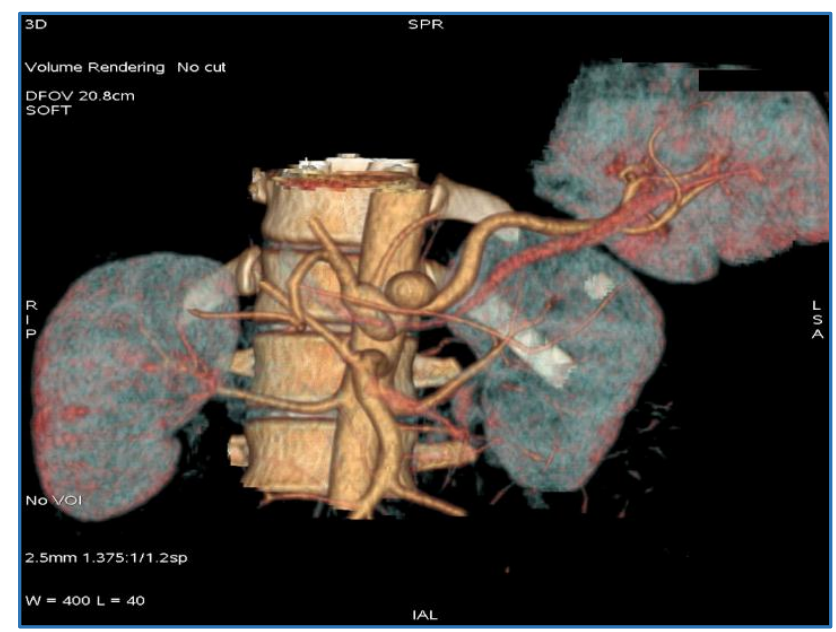

Figure 4. Volume Rendered Image-Pseudoaneurysm of Splenic Artery Sequelae to Acute Pancreatitis

\section{DISCUSSION}

Acute pancreatitis defined as an acute inflammatory process of the pancreas that may involve peripancreatic tissues and/or remote organ systems. ${ }^{[4]}$ Incidence of acute pancreatitis is increasing. Recognition of severe pancreatitis by clinical examination is unreliable.[5] Plasma levels of amylase and lipase enzymes as a diagnostic indicator have no role in the assessment of disease severity. The serum trypsinogen level may be useful as a predictive indicator, but it has not gained clinical acceptance because useful clinical assay is not currently available.[6] Many serum and urinary levels of enzymes and markers are available, but are of limited clinical use. ${ }^{[7-9]}$

Acute pancreatitis is divided into- 1) Interstitial oedematous pancreatitis and 2) Necrotising pancreatitis. Interstitial oedematous pancreatitis have diffuse or localised enlargement of the pancreas due to inflammatory oedema. CECT pancreatic parenchyma shows relatively homogeneous enhancement and inflammatory changes in peripancreatic fat in the form of haziness or mild stranding and sometimes peripancreatic fluid collection. Necrotising pancreatitis occurs due to necrosis of pancreatic parenchyma, peripancreatic tissue or both. CECT shows patchy nonenhancing area suggestive of parenchymal necrosis. In peripancreatic necrosis pancreas enhances normally on CECT, but the peripancreatic tissues develop necrosis. Pancreatic and peripancreatic necrosis can remain sterile or become infected. Various local and systemic complications can occur due to pancreatitis. Local complications can be parenchymal necrosis, could be sterile or infected, pancreatic fluid collections (Pseudocysts), involvement of contiguous organs, vascular complications or obstructive jaundice.[10]

Main purpose of CT in acute pancreatitis is to assess the severity of disease and categories patients who need hospitalisation and intensive care management. Balthazar $\mathrm{E}^{[2]}$ developed a grading system for pancreatitis as CT severity index to determine the severity of acute pancreatitis. Modified CT severity index correlates more closely with patient outcome measures than the CT severity index.[11] CT indices more accurately diagnose clinically severe disease and better correlate with the need for intervention and pancreatic infection. Modified CTSI differs from CTSI in two important aspects. It includes points for extrapancreatic complications such as pleural effusion, ascites, vascular or GI complications which received an extra 2 points on MCTSI scoring as compared to CTSI. MCTSI has a simpler quantification of amount of necrosis as $<30 \%$ or $>30 \%$ only, which is easier to apply. In MCTSI scoring points are given in increments of 2 , hence only even scores can be given. ${ }^{[3]}$

We observed that there is no association between age and gender with severity of pancreatitis. The maximum patients were in the age group of 25 to 35 years (33.3\%). The minimum age of patients was 5 years and maximum was 70 years. In $80 \%$ patients we observed alcohol as aetiology for pancreatitis, which is preventable cause. Percentage of necrosis and severity of pancreatitis is higher with alcoholic pancreatitis. Mendez GJ et al[12] also observed alcohol as a major cause of pancreatitis.

Pancreatic necrosis seen as focal non-enhancing low attenuation area in pancreatic parenchyma was noted in 20 patients (Figure 1). Sixteen had $\leq 30 \%$ of necrosis and four had $>30 \%$ of necrosis. Glandular necrosis is an important determinant of prognosis. Clinical and biochemical assessment cannot detect the extent of necrosis. CT scan very well delineate the amount of parenchymal damage. Bollen $\mathrm{T}$ 
et al[1] identified necrosis in $18 \%$ of patients with acute pancreatitis and observed that necrosis almost always occurs within 48 hours after onset of symptoms. We have higher percentage of necrotising pancreatitis, because of late presentation. Association of pancreatic necrosis and organ failure with alcohol was reported by Easler JJ et al.[13]

Acute peripancreatic fluid collection occurs in interstitial oedematous pancreatitis. Pancreatic pseudocyst refers specifically to a fluid collection in the peripancreatic or intrapancreatic tissues surrounded by a well-defined wall and contains essentially no solid material. It is a delayed (usually $>4$ weeks) complication of interstitial oedematous pancreatitis and necrosis.[10] The most common site of extrapancreatic phlegmon is lesser sac (38\%) followed by left anterior pararenal space. These findings are consistent with previous literature.[14-17] We observed 29 patients with mild pancreatitis and 13 with severe pancreatitis. These observations are similar to the observations of Bollen $\mathrm{T}$ et al.[1] The extrapancreatic complications are the major cause of morbidity and mortality in pancreatitis. Chishty et al[18] in a study of 40 patients observed higher incidence of extrapancreatic complications. Thrombosis of pancreatic tributaries of the portal vein is a known complication of pancreatitis.[17] The splenic vein along the upper posterior order of the pancreas is occluded in $45 \%$ of patients with pancreatitis [Figure 2]. CT demonstrates the venous thrombosis as well as the presence of vascular collaterals. We had seven patients of splenic vein thrombosis and three had portal vein thrombosis.

Vascular complication of pancreatitis can lead to episodes of life-threatening haemorrhage due to arterial erosion and venous thrombosis due to the proteolytic effects of pancreatic enzymes on important pancreatic and peripancreatic vessels that can lead to frank haemorrhage or pseudoaneurysm formation. Splenic artery, pancreaticoduodenal and gastroduodenal arteries are most commonly involved in acute pancreatitis. Dynamic CT is the most useful modality for diagnosis of vascular complication with pancreatitis. It helps in diagnosis of pancreatitis and identify the fluid collection in patient with haemorrhage, determine the extent of inflammatory process and its proximity to important vascular structure. It also helps to diagnose the presence of haemorrhage on CT in a fluid collection due to increased attenuation of contents (130 HU) and can identify pseudoaneurysm formation seen as a transient vascular enhancement in a cystic pancreatic mass. [17]

We have noted pseudoaneurysm formation with thrombosis of the right hepatic artery [Figure 3], inferior pancreaticoduodenal and splenic artery. Out of 60 patients of pancreatitis, 13 suffered from vascular complications. Pseudocysts which are encapsulated collections of pancreatic fluid were seen in 29 patients and in 14 it was located in lesser sac. We observed higher incidence of pseudocysts as compared to others, which could be attributed to late presentation with severe disease.[19-22]

The CT scores were classified into 2, 4, 6, 8 and 10 according to the MCTSI. We further classified the scores into mild (Score 2 and 4), moderate (Score 6) and severe (Score 8 and 10). The maximum patients were seen with Score 2 and 4 category (48.3\%) and minimum (21.6\%) with Score 8 and 10 category. Similarly, most of the patients were of mild CT severity (48.3\%) and few patients had severe score.
Moderate pancreatitis was observed in $30 \%$ of patients. According to the study by Bollen $T,{ }^{[1]}$ the morphologic severity of pancreatitis was scored on the basis of MCTSI and observed minimum patients of severe acute pancreatitis. Most patients are of mild score in the study probably due to early use of CECT in diagnosis and increased rate of detection of early pancreatitis.

\section{CONCLUSION}

Contrast Enhanced Computed Tomography of abdomen in acute pancreatitis helps in differentiating between acute oedematous and necrotising pancreatitis. The MCTSI helps in evaluating the percentage of pancreatic necrosis and to predict the possibility of developing local and systemic complications.

Ultrasound of abdomen followed by CECT helps in early and better anatomical delineation of complications of acute pancreatitis. Depending upon the MCTSI grading, the treatment planning can be done more effectively and accurately. Mild-to-moderate acute pancreatitis with MCTSI scores upto six can be managed conservatively, whereas severe cases with MCTSI scores (8 - 10) require intensive care multidisciplinary approach.

\section{REFERENCES}

[1] Bollen T, Singh VK, Maurer R, et al. Comparative evaluation of the modified CT severity index and CT severity index in assessing severity of acute pancreatitis. AJR Am J Roentgenol 2011;197(2):38692.

[2] Balthazar EJ. Acute pancreatitis: assessment of severity with clinical and CT evaluation. Radiology 2002;223(3):603-13.

[3] Mortele KJ, Wiesner W, Intriere L, et al. A modified CT severity index for evaluating acute pancreatitis: improved correlation with patient outcome. Am J Roentgenol 2004;183(5):1261-5.

[4] Banks PA. Practice guidelines in acute pancreatitis. Am J Gastroenterol 1997;92(3):377-86.

[5] Dickson AP, Imrie CW. The incidence and prognosis of body wall ecchymosis in acute pancreatitis. Surg Gynaecol Obstet 1984;159(4):343-7.

[6] Corfield AP, Cooper MJ, Williamson RCN. Prediction of severity in acute pancreatitis: prospective comparison of three prognostic indices. Lancet 1985;326(8452):403-7.

[7] Sainio V, Puolakkainen P, Kempainnen E, et al. Serum trypsinogen-2 in the prediction of outcome in acute necrotizing pancreatitis. Scand J Gastroenterol 1996;31(8):818-24.

[8] Tenner S, Fernandez-del CC, Warshaw A, et al. Urinary Trypsinogen Activation Peptide (TAP) predicts severity in patients with acute pancreatitis. Int J Pancreatol 1997;21(2):105-10.

[9] Lankisch PG, Schirren CA, Otto J. Methemalbumin in acute pancreatitis: an evaluation of its prognostic value and comparison with multiple prognostic parameters. Am J Gastroenterol 1989;84(11):1391-5.

[10] Banks PA, Bollen TL, Dervenis C. Classification of acute pancreatitis-2012: revision of the Atlanta classification and definitions by international consensus. Gut 2013;62(1):102-11. 
[11] Triantopoulou C, Lytras D, Maniatis P, et al. Computed tomography versus Acute Physiology and Chronic Health Evaluation II score in predicting severity of acute pancreatitis: a prospective, comparative study with statistical evaluation. Pancreas 2007;35(3):23842.

[12] Mendez G, Isikott MB, Hill MC. CT of acute pancreatitis: interim assessment. AJR Am J Roentgenol 1980;135(3):463-9.

[13] Easler JJ, de-Madaria E, Nawaz H, et al. Patients with sentinel acute pancreatitis of alcoholic aetiology are at risk for organ failure and pancreatic necrosis: a dual centre experience. Pancreas 2016;45(7):997-1002.

[14] Siegelman SS, Copeland BE, Saba GP, et al. CT of fluid collections associated with pancreatitis. AJR Am J Roentgenol 1980;134(6):1121-32.

[15] Federle MP, Jeffrey RB, Crass RA, et al. Computed tomography of pancreatic abscesses. AJR Am J Roentgenol 1981;136(5):879-82.

[16] Sutton PA, Humes DJ, Purcell G, et al. The role of routine assays of serum amylase and lipase for the diagnosis of acute abdominal pain. Ann R Coll Surg Engl 2009;91(5):381-4.
[17] Vujic I. Vascular complications of pancreatitis. Radiological Clinics of North America 1989;27(1):8191.

[18] Chishty IA, Bari V, Pasha S, et al. Role of computed tomography in acute pancreatitis and its complications among age groups. Pak Med Assoc 2005;55(10):431-5.

[19] Gonzale P, Nagar B, Gorelick S. Pseudocyst formation in acute pancreatitis. AJR 1976;127:315-7.

[20] Ferrucci JT, Wittenberg J, Black EB, et al. Computed body tomography in chronic pancreatitis. Radiology 1979;130(1):175-82.

[21] Kolmannskog F, Schrumpf E, Bergan A, et al. Diagnostic value of computed tomography in chronic pancreatitis: a comparision with current diagnostic tests. Acta Radiological Diagnosis (Stockh) 1981;22(6):649-55.

[22] Luetmer PH, Stephens DH, Ward EM. Chronic pancreatitis: reassessment with current CT. Radiology 1989;171(2):353-7. 